\title{
New Current-Mode Integrated Ternary Min/Max Circuits without Constant Independent Current Sources
}

\author{
Mona Moradi, ${ }^{1}$ Reza Faghih Mirzaee, ${ }^{2}$ and Keivan $\mathrm{Navi}^{3}$ \\ ${ }^{1}$ Department of Computer Engineering, Islamic Azad University, Science and Research Branch, Tehran 1477893855, Iran \\ ${ }^{2}$ Department of Computer Engineering, Islamic Azad University, Shahr-e-Qods Branch, Tehran 37541-374, Iran \\ ${ }^{3}$ Faculty of Electrical and Computer Engineering, Shahid Beheshti University, G.C., Tehran 1983963113, Iran
}

Correspondence should be addressed to Mona Moradi; mo.moradi@srbiau.ac.ir

Received 9 February 2015; Revised 23 March 2015; Accepted 23 March 2015

Academic Editor: Ahmed El Wakil

Copyright (C) 2015 Mona Moradi et al. This is an open access article distributed under the Creative Commons Attribution License, which permits unrestricted use, distribution, and reproduction in any medium, provided the original work is properly cited.

\begin{abstract}
Novel designs of current-mode Ternary minimum (AND) and maximum (OR) are proposed in this paper based on Carbon NanoTube Field Effect Transistors (CNTFET). First, these Ternary operators are designed separately. Then, they are combined together in order to generate both outputs concurrently in an integrated design. This integration results in the elimination of common parts when both functions are required at the same time. The third proposed current-mode integrated circuit generates both ternary operators with the usage of only 30 transistors. The new designs are composed of three main parts: (1) the part which converts current to voltage; (2) threshold detectors; and (3) the parallel paths through which the output current flows. Unlike the previously presented structure, there is no need for any constant current source within the new designs. This elimination leads to less static power dissipation. The second proposed current-mode segregated Ternary minimum operates $43 \%$ faster and consumes $40 \%$ less power in comparison with a previously presented structure.
\end{abstract}

\section{Introduction}

In current-mode logic (CML), logic levels are represented by current levels. It has several advantages over voltage-mode logic (VML). The merits include (1) simple wiring of the currents, making linear sum operation easier and reducing the number of active devices [1]; (2) the ease of circuit expansion [2]; (3) the usage of the direction of current as the explanation of sign, which eliminates the requirement of sign bit [1]; (4) lower noise sensitivity [3]; (5) scaling and copying the currents with a simple current mirror circuit [4]; and, last but not least, (6) high-speed operation [4]. However, despite its several advantages, high static power consumption is the main drawback of CML.

Current-mode circuits are traditionally implemented by either bipolar (bipolar CML) or MOS (MCML) devices. MCML is preferred for mixed analog-digital signal environments due to high power consumption of bipolar transistors and higher supply noise immunity of MOS devices [3]. Although the latter has been the superior technology for implementing energy-efficient circuits for many years, their suitability in today's nanometer VLSI industry is gradually fading away. This is mainly because of several critical challenges of MOS devices in nanoranges such as very high leakage currents, high power density, large parametric variations, and decreased gate control [5]. To overcome these difficulties, Carbon NanoTube Field Effect Transistor (CNTFET) is considered as the most promising successor to the MOS technology in the near future. This is mainly because of its intrinsic similarities to current technology. In addition, it benefits from the same motility of both $n$ type and $p$-type CNTFET devices, high-speed operation, low power consumption, and the realization of desired threshold voltage, which is a great feature in multiple-valued logic (MVL) circuitry [6].

MVL is an approach that utilizes more than two logic levels, while Binary logic computations are based on two values ("0" and " 1 "). MVL circuits benefit from fewer interconnections and pinouts, less area dissipation, and higher parallel and serial communication rates [1]. MVL is also 
known as an alternative design technique to Binary circuits, where the amount of interconnections is becoming a serious challenge. The huge amount of wires inside today's nanoscale chips adds undesirable parasitic effects, reduces noise tolerance, dissipates more power, and restricts the routing and placement processes of logic elements [7-9]. The increment of logic levels can be considered as a solution to these difficulties and limitations. MVL circuits will play an important role in the next generation of electronic systems [1]. The most efficient MVL system, which leads to less product cost and complexity than Binary, is Ternary logic [7].

In this paper, new Ternary minimum (Logical AND) and maximum (Logical OR) circuits are proposed. They are based on CNTFET technology and CML design technique. They are fundamental operators in mathematical and logical components and computational units such as Arithmetic Logic Unit (ALU). Since both operators are required at the same time in most processors, their circuits are combined together to share the common parts and reduce transistorcount. The new circuits are based on mixed current and voltage logics. The usage of constant current source is avoided in the new designs. Therefore, power dissipation is reduced dramatically in comparison with the previously presented structure.

The rest of the paper is organized as follows: Section 2 reviews the previously presented current-mode MVL-based works in the literature. The transistor-level implementation of the previous design is also presented in this section. New current-mode Ternary Min/Max circuits are proposed in Sections 3 and 4. Simulation results and comparisons are brought in Section 5. Finally, Section 6 concludes the paper.

\section{Literature Review}

New minimum and maximum circuits have been presented in [10-12] for fuzzy logic. They are based on linear addition and subtraction of input currents, and they benefit from design simplicity and low transistor-count. Although it is apparently possible to use them in any MVL system, they have not been particularly designed for Ternary logic or any other digital systems. As a result, they fail to refresh and fortify incomplete signals. One of the major features of circuits in discrete systems is the ability of generating full-swing outputs in spite of non-full-swing input signals. Therefore, the employment of these fuzzy circuits [10-12] in digital systems is neither suitable nor practical.

Some current-mode structures have been previously presented in [13] for implementing signed Ternary functions. A comparable method has been used in [14] to design an unsigned Ternary circuit, which is the main objective in this paper. The previous Ternary maximum and minimum circuits, presented in [14], are shown in Figures 1(a) and 1(b), respectively. These structures utilize constant independent current sources as threshold detectors. In case the input current ( $a$ or $b$ ) is less than the constant one (the threshold), the rest of the current charges the gate capacitor of a $p$ type transistor, turning it off. If the amount of input current is more than the constant one, the transistor, whose gate capacitor is discharged, switches on. The transistor-level
TABLE 1: The truth table of Ternary minimum and maximum.

\begin{tabular}{ccccc}
\hline$a$ & $b$ & $\sum$ in & Minimum & Maximum \\
\hline 0 & 0 & 0 & 0 & 0 \\
0 & 1 & 1 & 0 & 1 \\
0 & 2 & 2 & 0 & 2 \\
1 & 0 & 1 & 0 & 1 \\
1 & 1 & 2 & 1 & 1 \\
1 & 2 & 3 & 1 & 2 \\
2 & 0 & 2 & 0 & 2 \\
2 & 1 & 3 & 1 & 2 \\
2 & 2 & 4 & 2 & 2 \\
\hline
\end{tabular}

implementation of constant independent current sources, presented in [15], is used in this paper for simulation purpose (Figure 1(c)).

In the unsigned Ternary logic, there are three positive logic values, $\{0,1,2\}_{3}$. They are represented in current-mode logic by different current levels. The unit current of $8 \mu \mathrm{A}$ is considered in this paper to represent logic value " 1 ". Therefore, the logic value " 2 " is implemented by $16 \mu \mathrm{A}$, while no current flows when logic value is " 0 ".

The final representation of the previously presented Ternary Min and Max are shown in Figure 2, where each transistor is marked with three values (numbers). They indicate the diameter of CNTs $\left(D_{\mathrm{CNT}}\right)$, the number of nanotubes under the gate terminal (Tube), and the pitch parameter. These designs require constant currents of $4 \mu \mathrm{A}, 8 \mu \mathrm{A}, 12 \mu \mathrm{A}$, and $28 \mu \mathrm{A}$ as threshold detectors, whose transistor-level implementations are included in the figures. In addition, variable input currents ( $a$ and $b$ ) are duplicated by current mirrors since two copies of them are needed in each circuit.

\section{The Proposed Current-Mode Segregated Ternary Operators (CSTO)}

Logical AND and OR are among the most essential and fundamental operators in digital electronics. They are, respectively, equal to the minimum and maximum mathematical functions regardless of what MVL system is used. Their function in Ternary logic is shown in Table 1. In this section, new Ternary Min and Max circuits are proposed separately. They are based on a technique where input currents are converted to voltage. Then, threshold detectors control the switching activity of the transistors, which are situated on the output paths. A unit of current $(8 \mu \mathrm{A})$ flows through each output path in case all of the related transistors are switched on. If the output value equals " 2 ", the currents of two different paths are joined in order to increase the amount of current to $16 \mu \mathrm{A}$.

In this section, the segregated strategy is followed. Then, the separated designs are combined together to achieve an integrated circuit in the next section.

3.1. The First Approach with a Current Mirror in the Last Part. The first proposed CML Ternary Min circuit is shown in Figure 3, where each transistor is marked with three values 


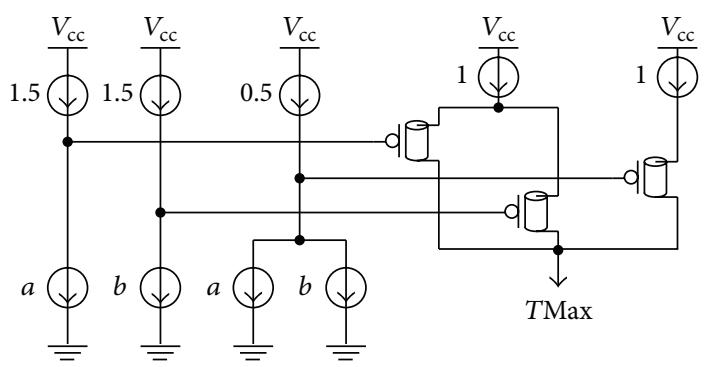

(a)

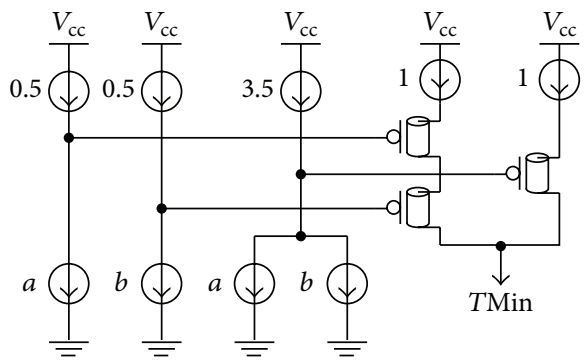

(b)

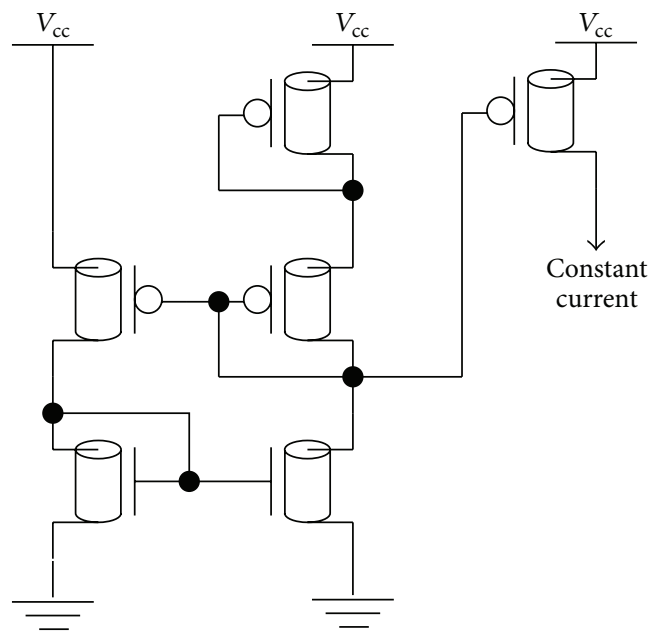

(c)

FIgURE 1: The previously presented circuits, (a) Ternary maximum [14], (b) Ternary minimum [14], (c) constant independent current source (transistor-level implementation) [15].

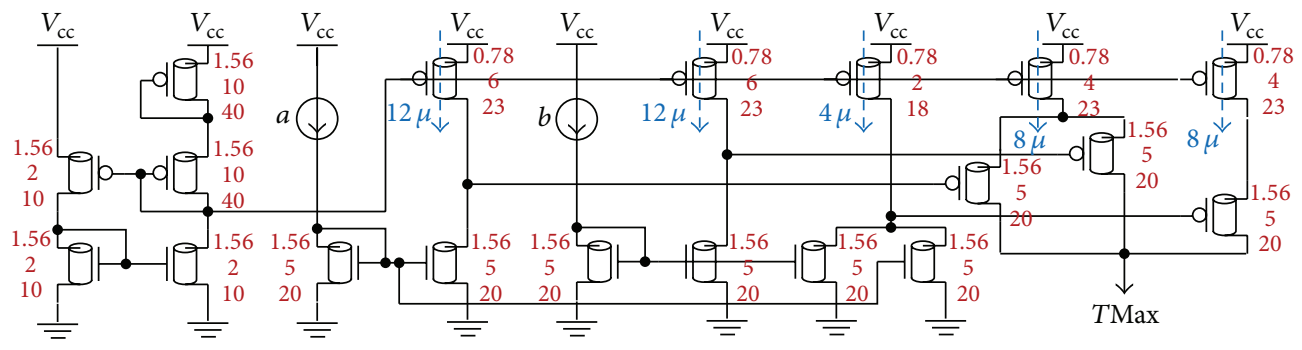

(a)

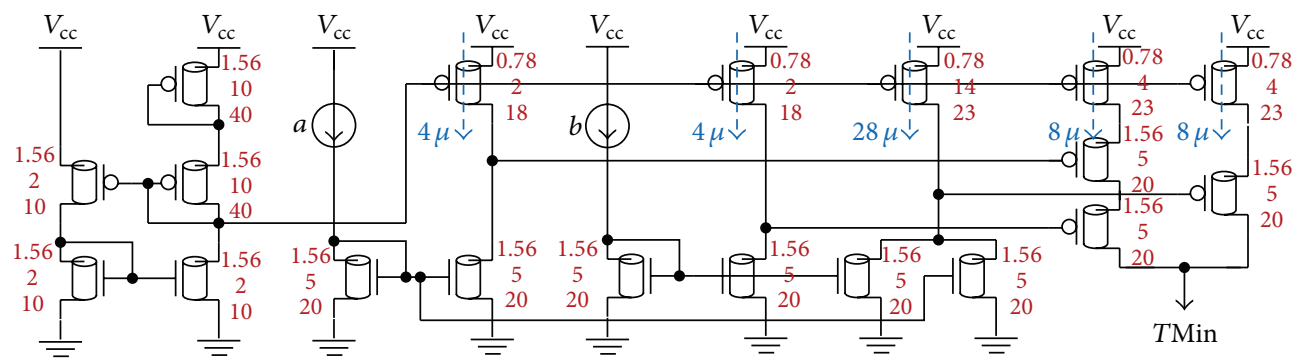

(b)

Figure 2: The previous current-mode circuits [14], (a) previous Ternary maximum (Previous TMax), (b) previous Ternary minimum (Previous TMin). 


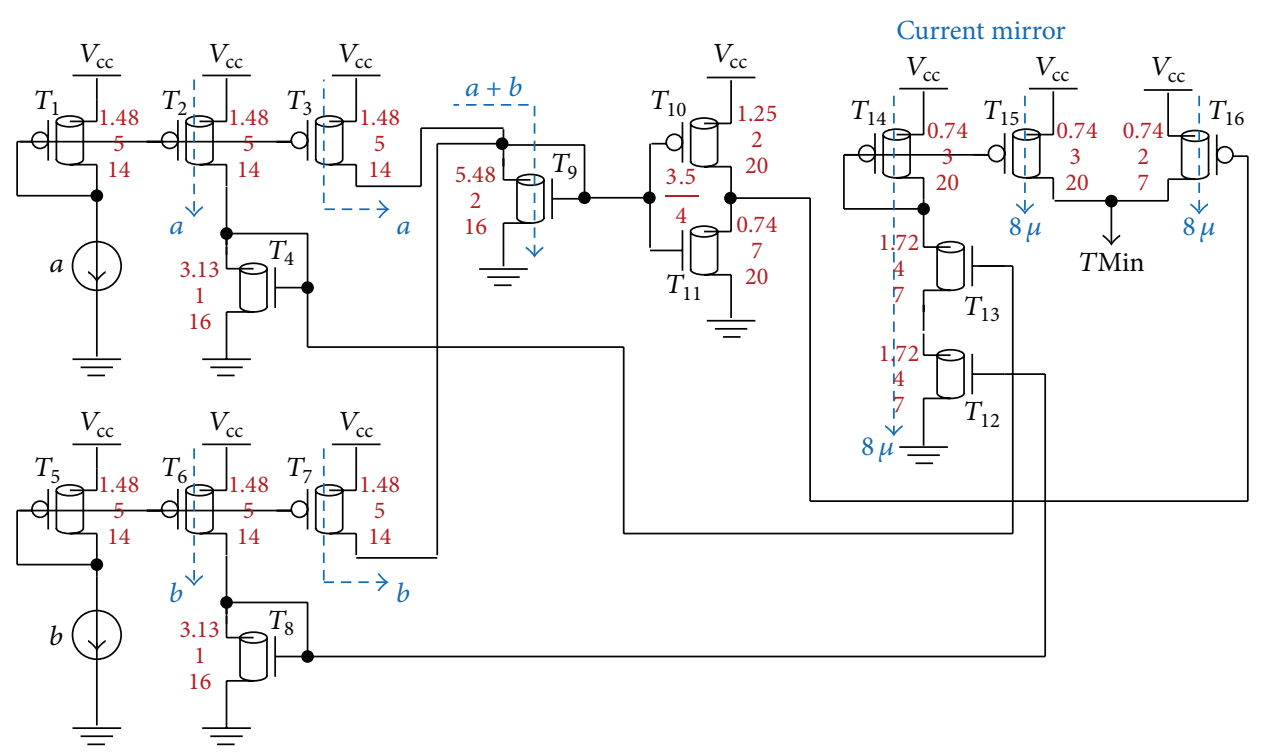

FIgURE 3: The first proposed Current-mode Segregated Ternary Min (CSTMin1) with the current mirror in the last part.

(numbers). As mentioned earlier, the numbers indicate the diameter of CNTs (in nanometer), the number of nanotubes under the gate terminal, and the pitch parameter (in nanometer). For example, there are eight nanotubes (Tubes $=8$ ) with the diameter of $1.48 \mathrm{~nm}\left(D_{\mathrm{CNT}}=1.48 \mathrm{~nm}\right)$ under the gate of $T_{1}$. The distance between the centers of two adjacent CNTs is also $14 \mathrm{~nm}($ Pitch $=14 \mathrm{~nm})$.

There are two input currents, $a$ and $b$. The proposed approach is on the basis of the sum of input currents. Unlike VML, it is as simple as connecting the wires ( $a$ and $b$ ) to achieve their linear addition in CML $(a+b)$. Then, the summation is converted to voltage by means of a resistor. A diode-connected transistor $\left(T_{9}\right)$ is used as a resistor to make this conversion possible. To obtain higher resistivity, channel $\left(L_{g}\right)$ and doped CNT source- $\left(L_{\mathrm{ss}}\right)$ and drain-side $\left(L_{\mathrm{dd}}\right)$ extension regions for this transistor have been lengthened by $90 \mathrm{~nm}$. The longer the channel is, the more it resists. This increment of channel length does not slow the operation because these converting transistors are not situated along the critical path of the cell.

The input currents ( $a$ and $b$ ), themselves, have to be converted to voltage as well. The transistors $T_{4}$ and $T_{8}$ are responsible for this purpose (for these two transistors: $L_{g}=$ $L_{\mathrm{ss}}=L_{\mathrm{dd}}=100 \mathrm{~nm}$ ). However, it is not feasible to take branches from the input currents due to the fact that the amount of current is divided. In CML, currents must be mirrored in case another copy it is required. In Figure 3, $T_{2}$ and $T_{3}\left(T_{6}\right.$ and $\left.T_{7}\right)$ duplicate the input current $a(b)$ twice. In order to have the exact copy, they must have the same dimensions as $T_{1}\left(T_{5}\right)$. This part of the circuit $\left(T_{1}\right.$ to $\left.T_{9}\right)$ is repeated in all of the proposed designs in this paper.

After the conversion, threshold detectors (TDs) control the switching activity of the rest of the transistors. Threshold detectors are in fact inverters with shifted voltage transfer characteristic (VTC) curves. There is only one TD in the first proposed Ternary Min circuit. The turning point of this TD is set 3.5/4 (Figure 3). It means that the output of this inverter is " 0 " only if the sum of input variables becomes the largest amount, four $\left(\sum\right.$ in $\left.=4\right)$.

The last part of the circuit, which determines the output value, is the most important one. It contains different paths, through which the output currents flow. The dimensions of the transistor(s) on a specific path have to be set properly so that exactly the unit current of $8 \mu \mathrm{A}$ flows in each path in case the related transistor(s) is/are switched on. The threshold voltage of $T_{12}$ and $T_{13}$ is set properly so that they switch on if $a \geq 1$ and $b \geq 1$, respectively. $T_{15}$ copies the current to the output node (Min). In case both input variables equal logic value " 2 " ( $\sum$ in $\left.=4\right)$, the threshold detector switches $T_{16}$ on, and another unit of current is added to the previous one. Hence, the total current reaches $16 \mu \mathrm{A}(\mathrm{Min}=$ " 2 ").

The same approach is used to design the first proposed CML Ternary Max circuit (Figure 4). The threshold voltage of $T_{16}$ is set "low." Therefore, it switches on as soon as $\sum$ in becomes greater than zero $\left(\sum\right.$ in $\left.\geq 1\right) . T_{17}$ lets the current flow until $\sum$ in reaches four $\left(\sum\right.$ in $\left.\leq 3\right)$. Consequently, the path is active in case $1 \leq \sum$ in $\leq 3 . T_{19}$ copies the same current to the output node (Max). There are two other parallel paths, constructed by the transistors $T_{20}$ and $T_{21}$. In case only one of the inputs is " 2 " $\left(2 \leq \sum\right.$ in $\left.\leq 3\right)$, a unit of current is added to the previous one (the copied one). If both are " 2 " $\left(\sum\right.$ in $\left.=4\right)$, the former path is inactive and the added transistors $\left(T_{20}\right.$ and $T_{21}$ ) generate $16 \mu \mathrm{A}$ current through two parallel paths.

\subsection{The Second Approach without the Usage of Current Mirror} in the Last Part. The second approach is almost the same as the first one except that the current mirror in the third part of the circuit is eliminated and the final currents are only generated by the $p$-type transistors. The second proposed CML Ternary Min and Max circuits are shown in Figures 5 and 6 , respectively. In Figure 5, $T_{16}$ and $T_{17}$ function exactly the same way as $T_{12}$ and $T_{13}$ in Figure 3 . However, the path 


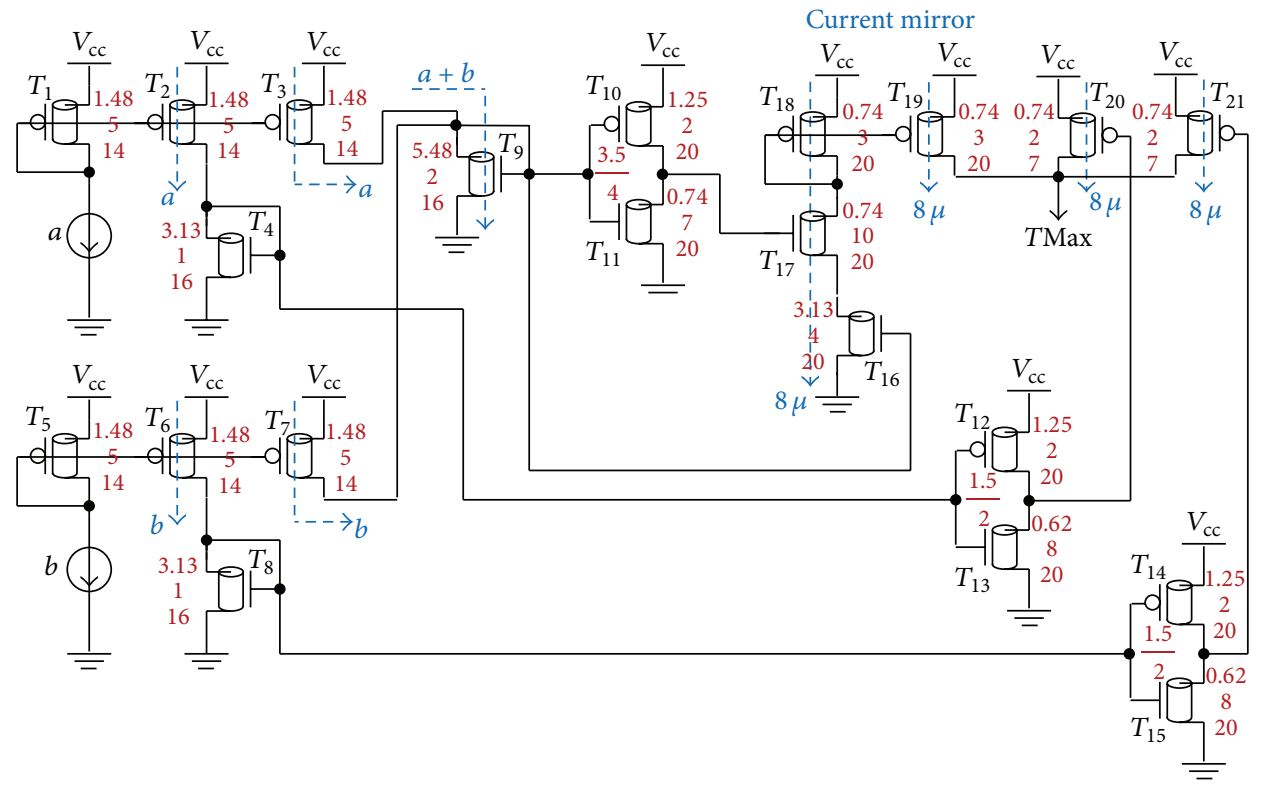

FIgURE 4: The first proposed Current-mode Segregated Ternary Max (CSTMax1) with the current mirror in the last part.

consists of only two transistors, one transistor fewer than the first approach, where current flows through two $n$-type transistors and a $p$-type one. The fewer transistors a path has, the more precise the amount of current is. In addition, the critical path shortens and the entire circuit becomes more robust.

In Figure 6, $T_{20}$ and $T_{21}$ are considered as the replacements of $T_{16}$ and $T_{17}$ in Figure 4. They let the current flow in case $1 \leq \sum$ in $\leq 3$. Although it is possible to connect the sum of input currents directly to the gate of $T_{21}$, it increases robustness if the activity of $T_{21}$ is controlled by a TD circuit. The full-swing output signal of a TD makes the following transistor switch on (or off) completely. In addition, there is no need to set the threshold voltage of this transistor precisely. It could have the same dimensions as $T_{20}$. It results in a simpler transistor sizing procedure as well. It is worth mentioning here that the threshold voltage of the CNTFET is an inverse function of $D_{\mathrm{CNT}}[16]$.

\section{The Proposed Current-Mode Integrated Ternary Operators (CITO)}

In some applications such as within the Arithmetic Logic Unit (ALU), both operators (AND and OR) are required at the same time. In this section, the previous segregated circuits are combined together in order to eliminate the common parts. The first proposed integrated circuit is shown in Figure 7. It is in fact the combination of CSTMin1 (Figure 3) and CSTMaxl (Figure 4), unless TDs are employed extensively to have a robust and accurate design. The common parts are the transistors which make the current to voltage conversion happen $\left(T_{1}\right.$ to $\left.T_{9}\right)$ and the inverter with the turning point of $3.5 / 4$.

It is also possible to share the $n$-type transistors $\left(T_{28} / T_{29}\right.$ and $\left.T_{33} / T_{34}\right)$. It leads us to the second integrated design
(Figure 8). While the Min circuit remains unchanged, the functionality of the Max circuit is summarized as follows (though a set of conditions):

(1) If $a \geq 1$ and $b \geq 1: T_{26}$ and $T_{27}$ switch on. A unit of current is copied to the both outputs (Min and Max).

(2) If $\sum$ in $\geq 3: T_{40}$ switches on. Another unit of current is added.

(3) If $a=$ "0" and $b \geq 1 \mid b=$ " 0 " and $a \geq 1$ : either $\left(T_{35}\right.$ and $\left.T_{36}\right)$ or $\left(T_{37}\right.$ and $\left.T_{38}\right)$ are $\mathrm{ON}$ and a unit of current flows.

(4) If $a=$ " 0 " and $b=$ " 2 " $\mid a=$ " 2 " and $b=$ "0": either $\left(T_{31}\right.$ and $\left.T_{32}\right)$ or $\left(T_{33}\right.$ and $\left.T_{34}\right)$ are $\mathrm{ON}$ and another unit of current is added to (3). The output becomes " 2 ".

The last design (Figure 9) is the combination of the second proposed segregated designs, CSTMin2 (Figure 5) and CSTMax2 (Figure 6). It has the fewest number of transistors among the integrated designs. It generates both outputs with the usage of only 30 transistors. Figure 10 shows the signal waveforms of this last design. The input pattern includes 72 transitions. The output signals are generated at the same time, and their values are exactly $0 \mu \mathrm{A}, 8 \mu \mathrm{A}$, and $16 \mu \mathrm{A}$, representing logic values " 0 ", " 1 ", and " 2 ".

\section{Simulation Results}

All of the proposed designs as well as the previous ones (Figure 2) are simulated with Synopsys HSPICE and $32 \mathrm{~nm}$ CNTFET technology file $[17,18]$. The simulations are carried out in $1 \mathrm{~V}$ power supply with $1 \mathrm{GHz}$ operating frequency at room temperature. The complete input pattern including 72 transitions (Figure 10) is fed to the circuits to reveal the worst-case delay. During all transitions, the average power consumption is measured. The energy consumption 


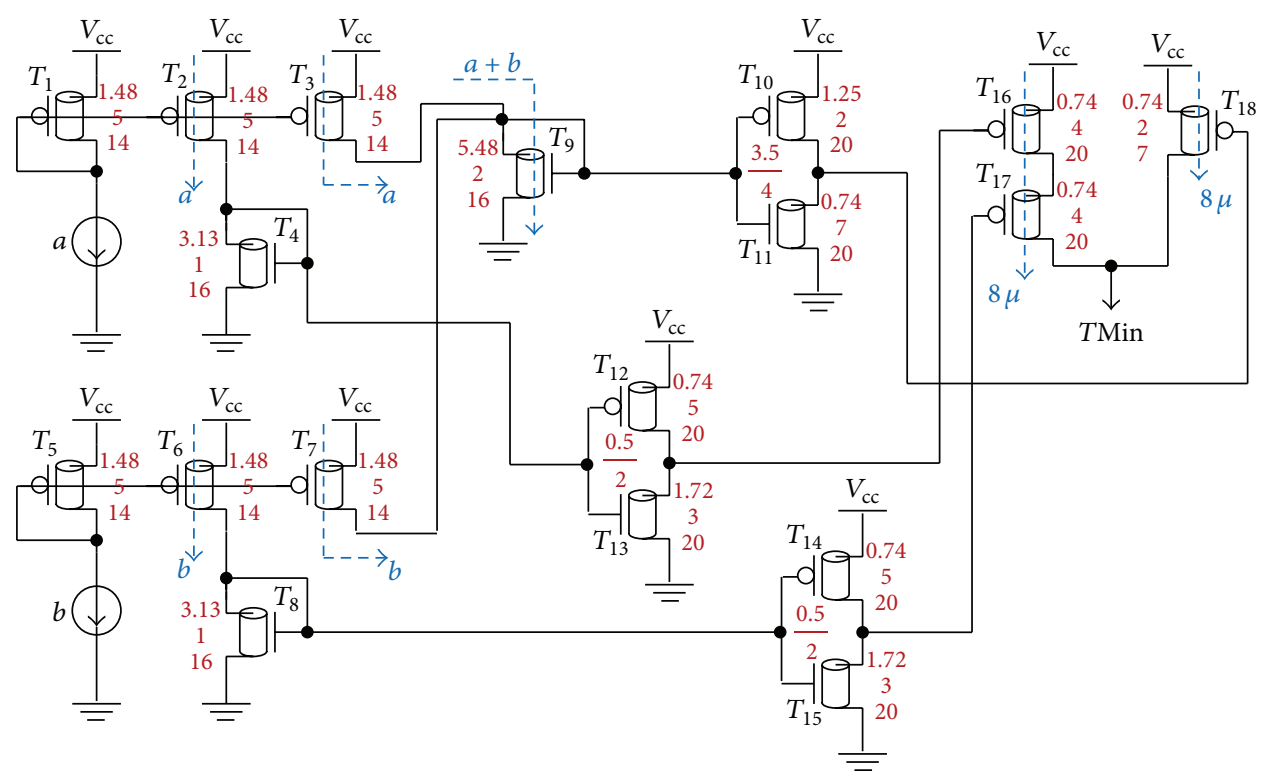

FIGURE 5: The second proposed Current-mode Segregated Ternary Min (CSTMin2) without the usage of current mirror in the last part.

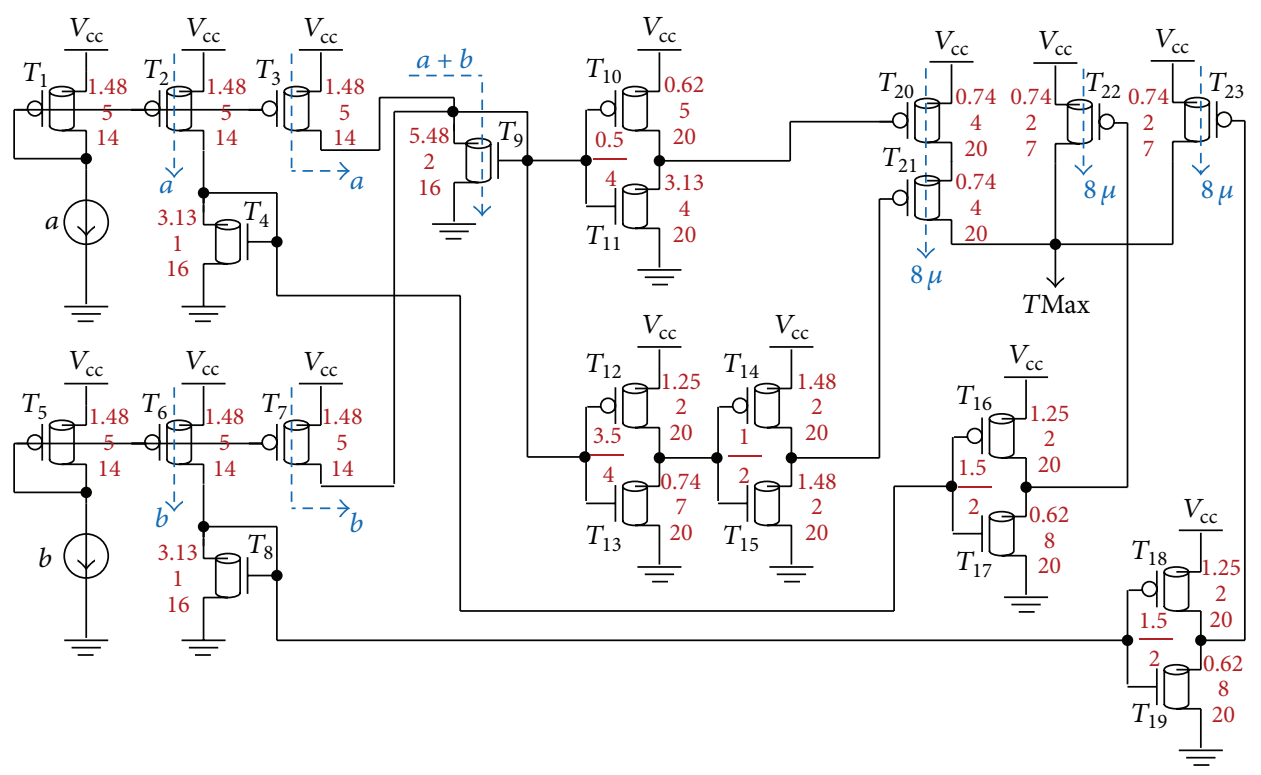

FIGURE 6: The second proposed Current-mode Segregated Ternary Max (CSTMax2) without the usage of current mirror in the last part.

(also known as PDP) is the multiplication of the maximum delay time and the average power consumption [16]. The average static power is also measured while the inputs are kept unchanged. The entire nine possible different input patterns (Table 1) are fed to the circuits to measure stand-by power dissipation. The average amount is reported as the static power.

The simulation results are exhibited in Table 2. In general, Max circuits consume more power than Min ones do. This is mainly because the output function is more likely to be " 0 " in Ternary AND than Ternary OR (Table 1). To talk literally, when applying a random input pattern, the probability of Ternary maximum being " 0 " is only $1 / 9$, while Ternary minimum has the same value in most cases (5/9). Therefore, less current flows in a Ternary Min circuit.

The proposed designs operate far more efficient than the previous ones. For example, the first and the second proposed segregated Ternary Min cells (CSTMin1 and CSTMin2) consume $25.64 \mu \mathrm{W}(35 \%)$ and $29.37 \mu \mathrm{W}(40 \%)$ less power than the previous one, respectively. Higher power consumption is mainly because of the constant current sources in the previous circuits. New designs are even faster, resulting in a great reduction in terms of PDP. Moreover, the second segregated design technique leads to less power consumption and higher efficiency due to the elimination of a few transistors and a parallel path. For instance, CSTMin2 operates 


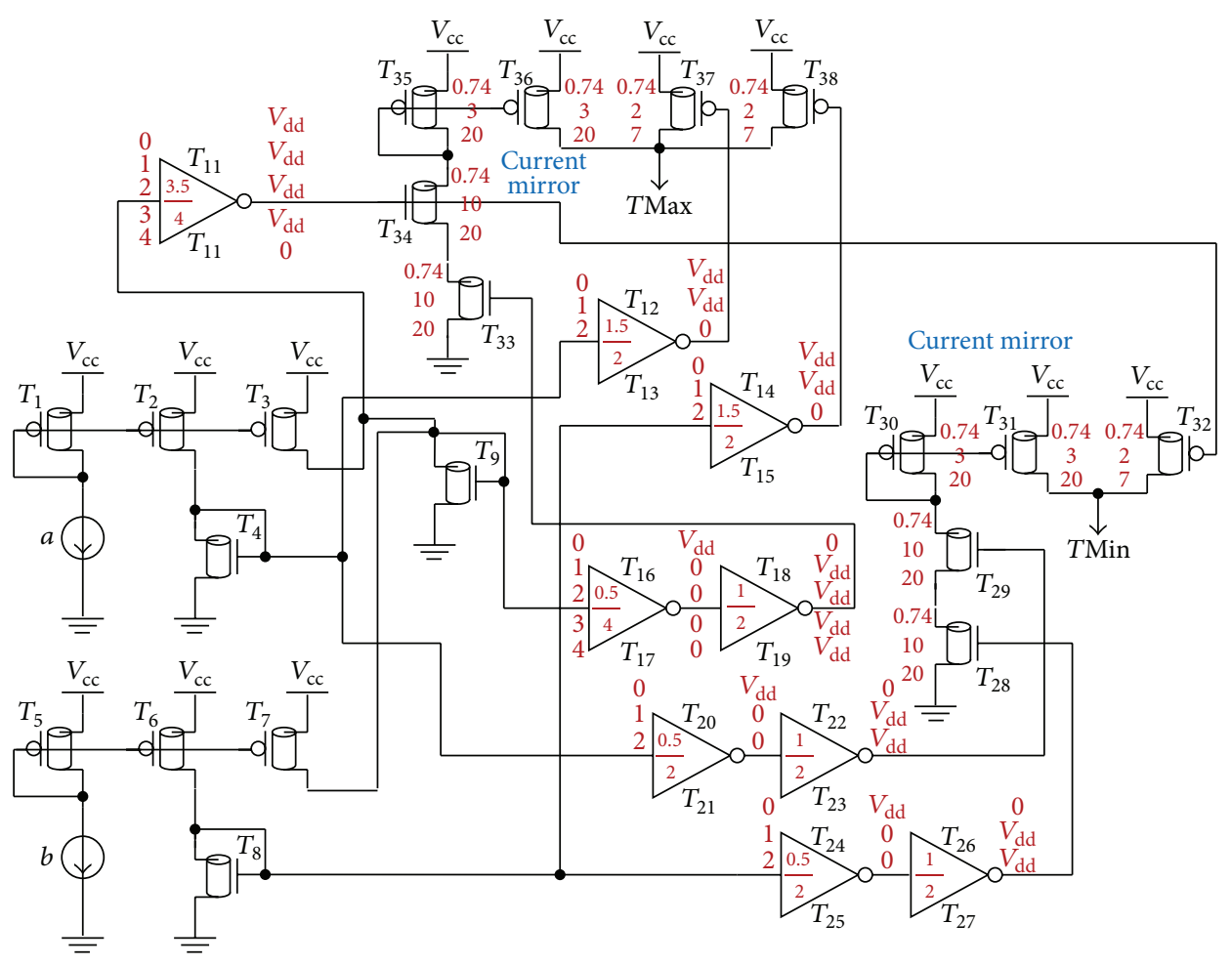

FIGURE 7: The first proposed Current-mode Integrated Ternary Min and Max (CITMin/Maxl) with the current mirrors in the last parts.

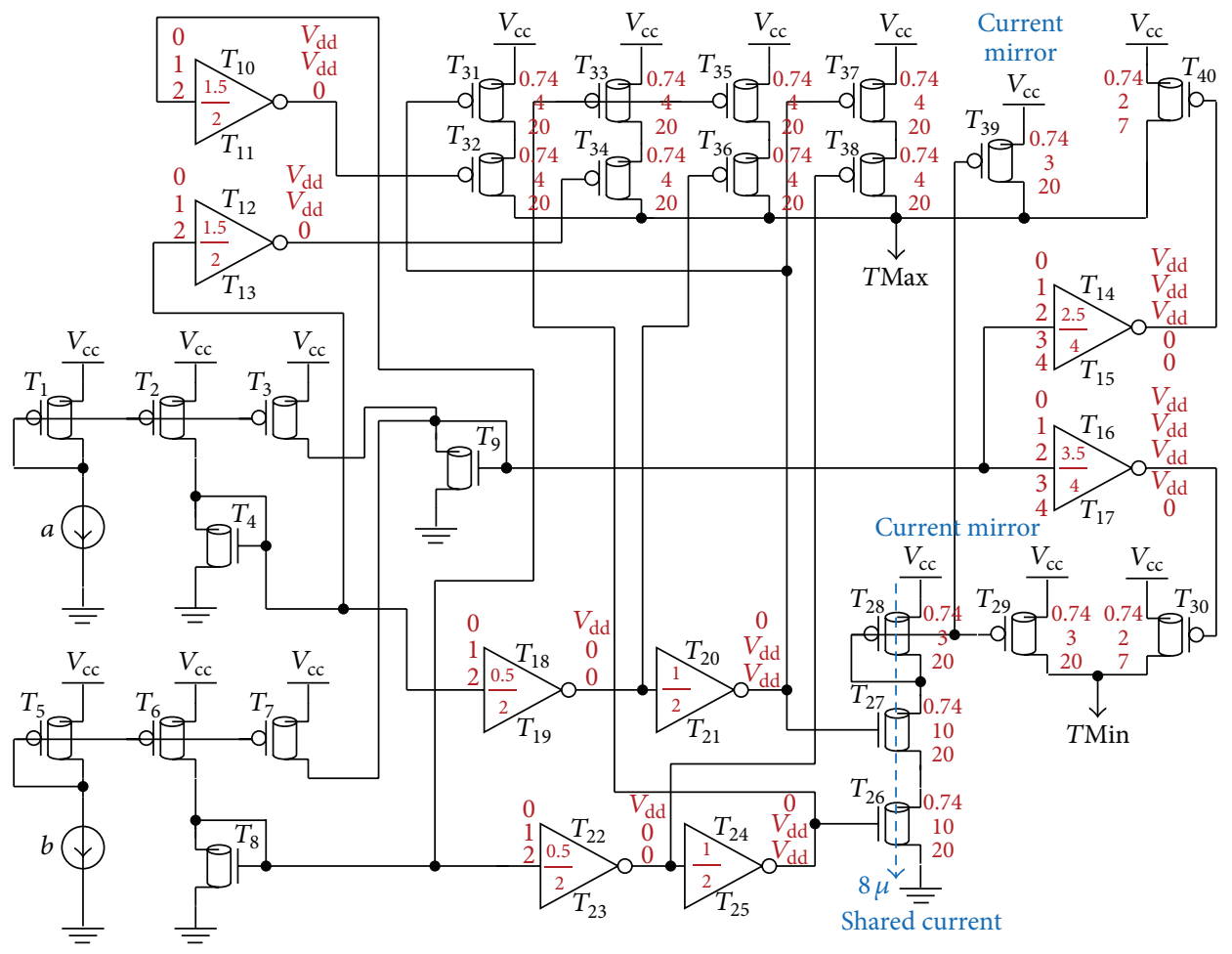

FIgURE 8: The second proposed Current-mode Integrated Ternary Min and Max (CITMin/Max2) with a shared current and the current mirrors in the last parts. 
TABLE 2: The initial simulation results.

\begin{tabular}{|c|c|c|c|c|c|c|}
\hline Designs & $\begin{array}{l}\text { Delay } \\
\text { (psec) } \\
\text { TMin }\end{array}$ & $\begin{array}{l}\text { Delay } \\
\text { (psec) } \\
\text { TMax }\end{array}$ & $\begin{array}{c}\text { Average } \\
\text { power } \\
(\mu \mathrm{W})\end{array}$ & $\operatorname{PDP}(\mathrm{fJ})$ & $\begin{array}{l}\text { Static } \\
\text { power } \\
(\mu \mathrm{W})\end{array}$ & $\begin{array}{l}\text { Number of } \\
\text { transistors }\end{array}$ \\
\hline Previous TMin & 19.64 & - & 72.56 & 1.42 & 72.94 & 19 \\
\hline CSTMin1 & 14.23 & - & 46.92 & 0.66 & 47.52 & 16 \\
\hline CSTMin2 & 11.17 & - & 43.19 & 0.48 & 43.71 & 18 \\
\hline Previous TMax & - & 14.93 & 75.62 & 1.12 & 76.06 & 19 \\
\hline CSTMaxl & - & 13.60 & 56.73 & 0.77 & 57.51 & 21 \\
\hline CSTMax2 & - & 13.71 & 50.40 & 0.69 & 51.09 & 23 \\
\hline CITMin/Max1 & 18.48 & 20.68 & 65.06 & 1.34 & 65.69 & 38 \\
\hline CITMin/Max2 & 18.11 & 21.38 & 58.53 & 1.25 & 59.06 & 40 \\
\hline CITMin/Max3 & 17.57 & 18.82 & 54.84 & 1.03 & 55.55 & 30 \\
\hline
\end{tabular}

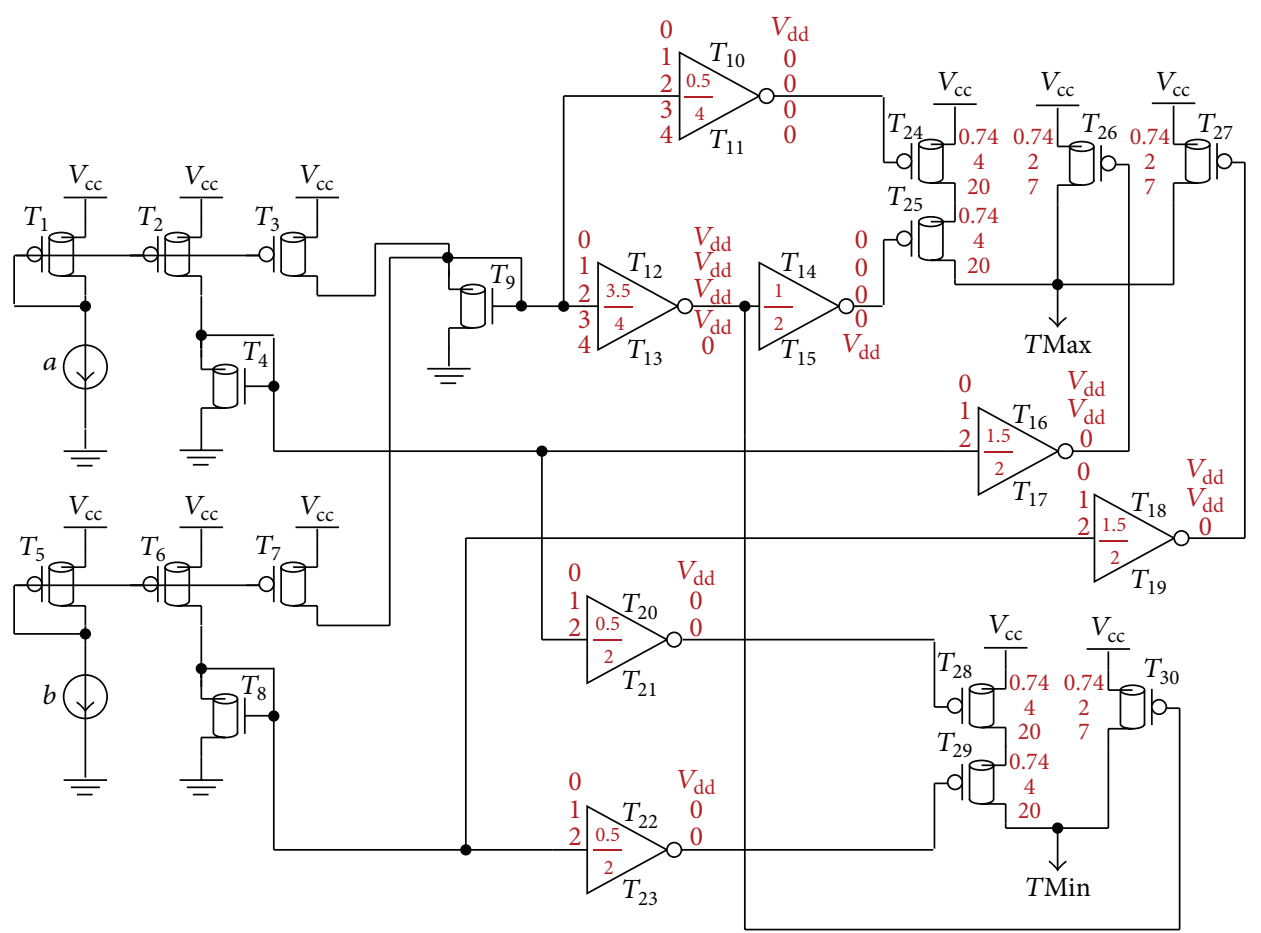

FIGURE 9: The third proposed Current-mode Integrated Ternary Min and Max (CITMin/Max3) without the usage of current mirrors in the last parts.

$21 \%$ faster, consumes $8 \%$ less power, and has 35\% higher performance (considering PDP) than CSTMin1. Eventually, the third integrated circuit (CITMin/Max3) has the highest performance in terms of energy. It generates both outputs with the fewest transistors needed.

Sensitivity to the variation of temperature is put under examination for the previous and some of the proposed designs. The amount of energy consumption (PDP) versus a wide range of ambient temperatures, from $0^{\circ} \mathrm{C}$ to $70^{\circ} \mathrm{C}$, is plotted in Figure 11. The proposed designs show insignificant sensitivity to temperature variations.

One major advantage of CML over VML is that fan-out circuits do not cause speed degradation and performance loss for the current-mode circuits. This is due to the way that fanout circuits are connected to a current-mode circuit. Instead of a direct connection, output currents are mirrored to the new branches (Figure 12). To test this capability, simulations are redone twice more. First, only the output load transistor is added to the proposed circuits. Then, four copies of the output current are also included in the examination. As it is demonstrated in Table 3, the existence of the output load transistor and the connection of the fan-out circuits do not increase cell delay. They do not add extra load to the output node. This is exactly in contrast with VML in which as the output load increases, voltage-mode circuits operate slower $[6,16]$. 
TABLE 3: Delay parameter of the proposed designs versus the output load(s).

\begin{tabular}{|c|c|c|c|c|c|c|}
\hline \multirow[b]{2}{*}{ Designs } & \multicolumn{2}{|c|}{ Without any output loads } & \multicolumn{2}{|c|}{ With the output load transistor } & \multicolumn{2}{|c|}{$\begin{array}{l}\text { With the output load transistor and } \\
4 \text { copies of the output current }\end{array}$} \\
\hline & $\begin{array}{c}\text { Delay (psec) } \\
\text { TMin }\end{array}$ & $\begin{array}{c}\text { Delay (psec) } \\
\text { TMax }\end{array}$ & $\begin{array}{l}\text { Delay } \\
\text { (psec) } \\
\text { TMin }\end{array}$ & Delay (psec) TMax & $\begin{array}{c}\text { Delay (psec) } \\
\text { TMin }\end{array}$ & $\begin{array}{c}\text { Delay (psec) } \\
\text { TMax }\end{array}$ \\
\hline CSTMinl & 14.23 & - & 14.44 & - & 14.40 & - \\
\hline CSTMin2 & 11.17 & - & 13.45 & - & 13.34 & - \\
\hline CSTMax1 & - & 13.60 & - & 13.79 & - & 13.80 \\
\hline CSTMax2 & - & 13.71 & - & 14.03 & - & 13.99 \\
\hline CITMin/Maxl & 18.48 & 20.68 & 18.81 & 21.31 & 18.76 & 21.20 \\
\hline CITMin/Max2 & 18.11 & 21.38 & 18.46 & 21.72 & 18.53 & 22.08 \\
\hline CITMin/Max3 & 17.57 & 18.82 & 17.76 & 19.27 & 17.71 & 19.33 \\
\hline
\end{tabular}
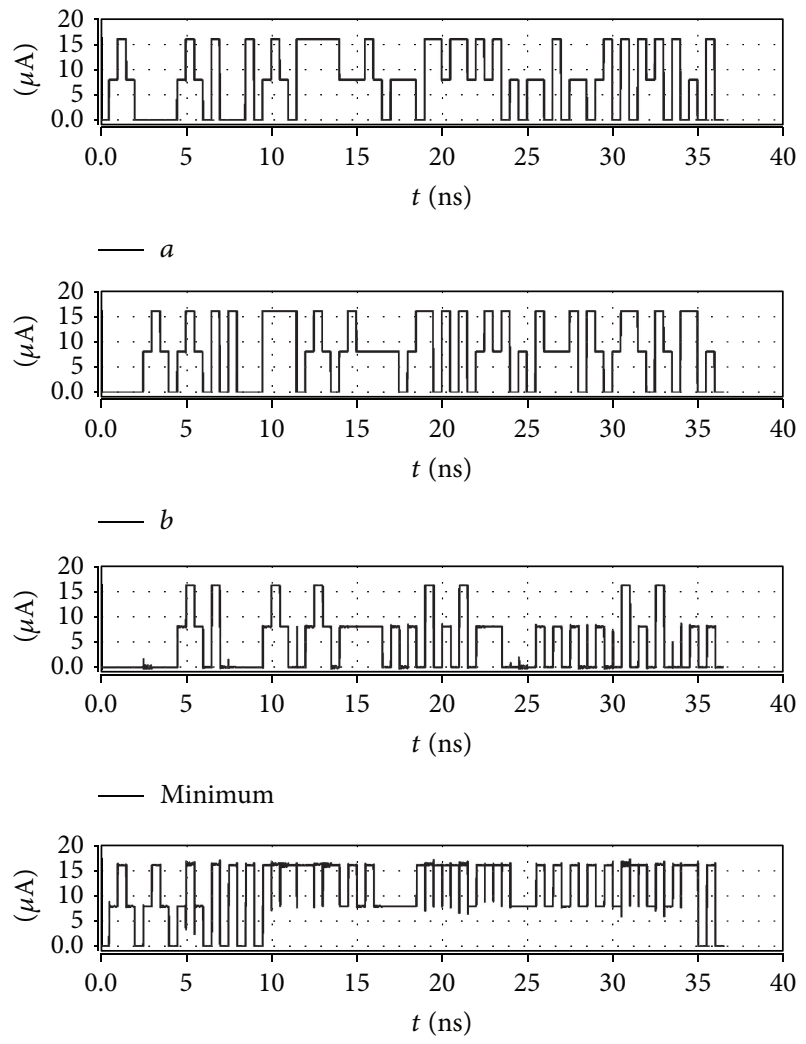

- Maximum

FIGURE 10: Input and output waveforms of CITMin/Max3.

Finally, as it was mentioned in Section 2, digital circuits must have the ability of refreshing incomplete signals. This is an absolute fact in all discrete MVL systems. Otherwise, the signal offset intensifies and propagates within the whole system and it causes information loss eventually. Figure 13 shows how the circuit CITMin/Max3 restores an incomplete input signal. This is the ability the fuzzy circuits like the ones presented in [10-12] do not have. As a result, they are not appropriate for any digital systems such as Ternary.

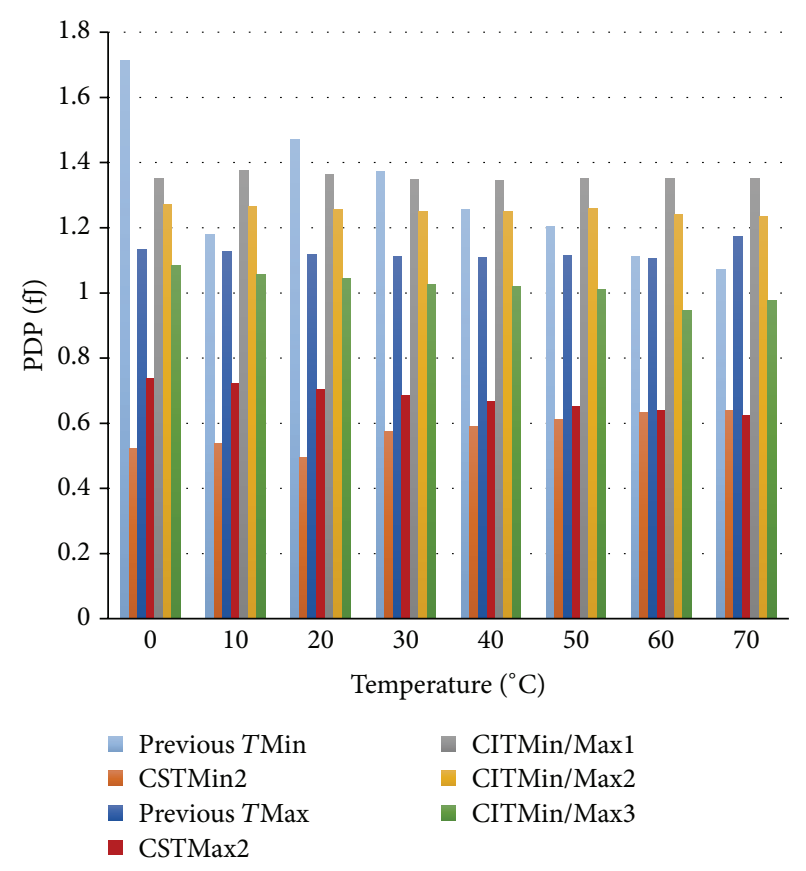

FIGURE 11: PDP versus temperature.

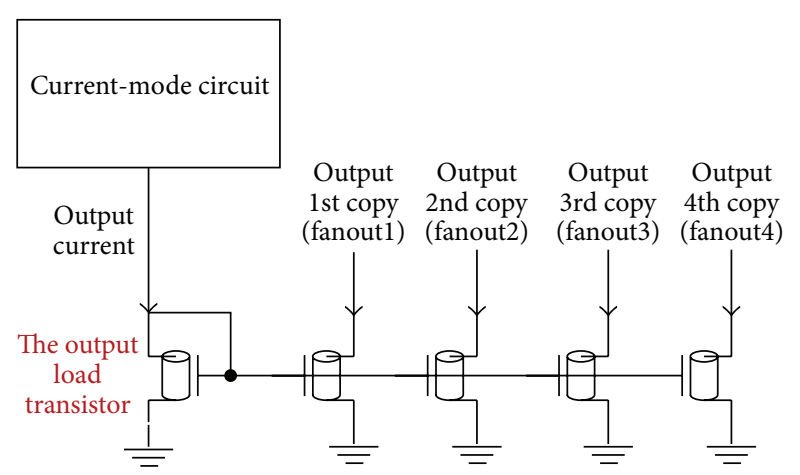

FIgURE 12: The output current is mirrored in CML to be connected to the fan-out circuits. 


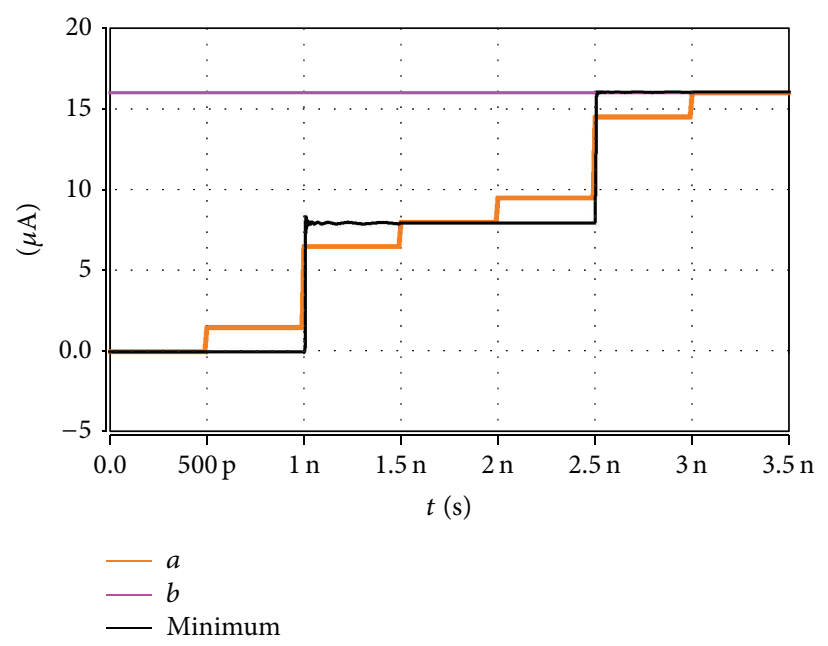

(a)

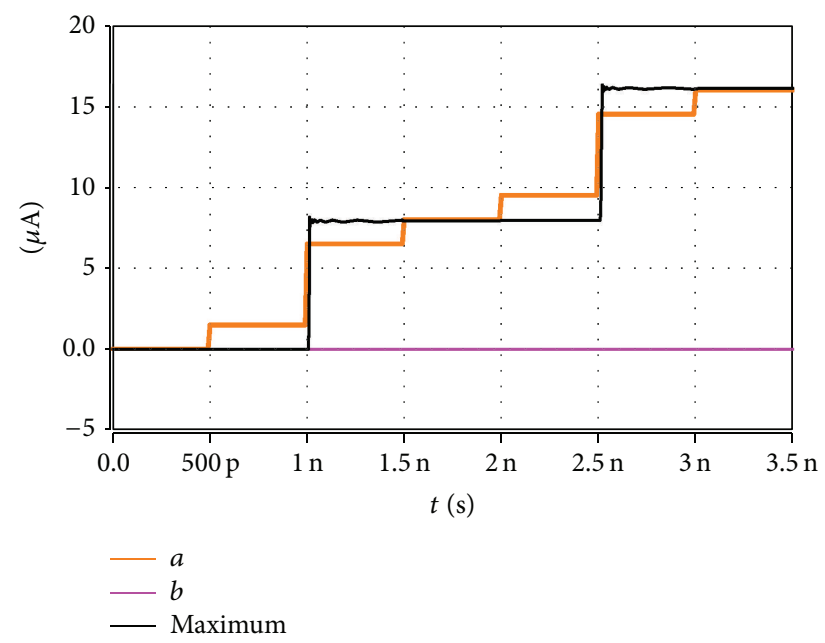

(b)

FIgURE 13: Signal restoration in CITMin/Max3, (a) Ternary minimum, (b) Ternary maximum.

\section{Conclusion}

In this paper, novel designs of current-mode Ternary Min and Max have been proposed. The new designs are based on mixed current and voltage logics, resulting in the elimination of constant independent current sources. Their elimination leads to higher performance for the proposed cells in comparison with the ones use constant current sources extensively as threshold detectors. The proposed CSTMin2 has approximately $66 \%$ higher efficiency than the previously presented design in terms of PDP. This paper also shows that, unlike VML, fan-out circuits have almost no effect on the delay parameter. Furthermore, Min circuits generally consume less power than the Max ones. This is due to the fact that the Ternary Min function is " 0 " in more input patterns than the Ternary Max one. For example, CSTMin 2 consumes $7.21 \mu \mathrm{W}$ less power than CSTMax2. Finally, the common parts could be easily integrated in order to combine two different circuits in CML. The integrated designs produce both Ternary minimum and maximum functions with almost the same speed.

\section{Conflict of Interests}

The authors declare that there is no conflict of interests regarding the publication of this paper.

\section{References}

[1] E. Dubrova, "Multiple-valued logic in VLSI: challenges and opportunities," in Proceedings of the 17th NORCHIP Seminar (NORCHIP '99), pp. 340-350, Oslo, Norway, November 1999.

[2] A. Kazeminejad, K. Navi, and D. Etiemble, "CML current mode full adders for 2.5-V power supply," in Proceedings of the 24th International Symposium on Multiple-Valued Logic, pp. 10-14, May 1994.

[3] Y. Delican and T. Yildirim, "High performance 8-bit mux based multiplier design using MOS current mode logic," in
Proceedings of the 7th International Conference on Electrical and Electronics Engineering (ELECO '11), pp. 89-93, December 2011.

[4] T. Temel and A. Morgul, "Multi-valued logic function implementation with novel current-mode logic gates," in Proceedings of the IEEE International Symposium on Circuits and Systems (ISCAS '02), vol. 1, pp. 881-884, 2002.

[5] S. Lin, Y.-B. Kim, F. Lombardi, and Y. J. Lee, "A new SRAM cell design using CNTFETs," in Proceedings of the International SoC Design Conference, pp. 168-171, November 2008.

[6] R. F. Mirzaee, M. H. Moaiyeri, M. Maleknejad, K. Navi, and O. Hashemipour, "Dramatically low-transistor-count high-speed ternary adders," in Proceedings of the IEEE 43rd International Symposium on Multiple-Valued Logic (ISMVL '13), pp. 170-175, May 2013.

[7] S. L. Hurst, "Multiple-valued logic, its status and its future," IEEE Transactions on Computers, vol. 33, no. 12, pp. 1160-1179, 1984.

[8] T. Sakurai, "Perspectives on power-aware electronics," in Proceedings of the IEEE International Solid-State Circuits Conference, Digest of Technical Papers (ISSCC '03), vol. 1, pp. 26-29, San Francisco, Calif, USA, February 2003.

[9] E. Özer, R. Sendag, and D. Gregg, "Multiple-valued logic buses for reducing bus energy in low-power systems," IEE Proceedings-Computers and Digital Techniques, vol. 153, no. 4, pp. 270-282, 2006.

[10] B. Mesgarzadeh, "A CMOS implementation of current-mode min-max circuits and a sample fuzzy application," in Proceedings of the IEEE International Conference on Fuzzy Systems, pp. 941-946, July 2004.

[11] G. Yosefi, S. Mirzakouchaki, and S. Neda, "Design of new CMOS current mode min and max circuits for FLC chip applications," in Proceedings of the European Conference on Circuit Theory and Design (ECCTD '09), pp. 89-92, IEEE, Antalya, Turkey, August 2009.

[12] T. Temel and A. Morgul, "Implementation of multi-valued logic gates using full current-mode CMOS circuits," Analog Integrated Circuits and Signal Processing, vol. 39, no. 2, pp. 191204, 2004. 
[13] J. Shen, X. Chen, and m. Yao, "Design of symmetric ternary current-mode CMOS circuits," Journal of Electronics, vol. 14, no. 4, pp. 336-344, 1997.

[14] W. Xunwei, D. Xiaowei, and Y. Shiyan, "Design of ternary current-mode CMOS circuits based on switch-signal theory," Journal of Electronics, vol. 10, no. 3, pp. 193-202, 1993.

[15] Q. N. Zhou, M. Y. Yu, and Y. Z. Ye, "On-chip voltage down converter with precision CMOS current source for VLSI chip," in Proceedings of the IEEE Conference on Electron Devices and Solid-State Circuits (EDSSC '05), pp. 375-378, December 2005.

[16] R. F. Mirzaee, K. Navi, and N. Bagherzadeh, "High-efficient circuits for ternary addition," VLSI Design, vol. 2014, Article ID 534587, 15 pages, 2014.

[17] J. Deng and H.-S. P. Wong, "A compact SPICE model for carbonnanotube field-effect transistors including nonidealities and its application-part I: model of the intrinsic channel region," IEEE Transactions on Electron Devices, vol. 54, no. 12, pp. 3186-3194, 2007.

[18] J. Deng and H.-S. P. Wong, "A compact SPICE model for carbonnanotube field-effect transistors including nonidealities and its application. Part II. Full device model and circuit performance benchmarking," IEEE Transactions on Electron Devices, vol. 54, no. 12, pp. 3195-3205, 2007. 

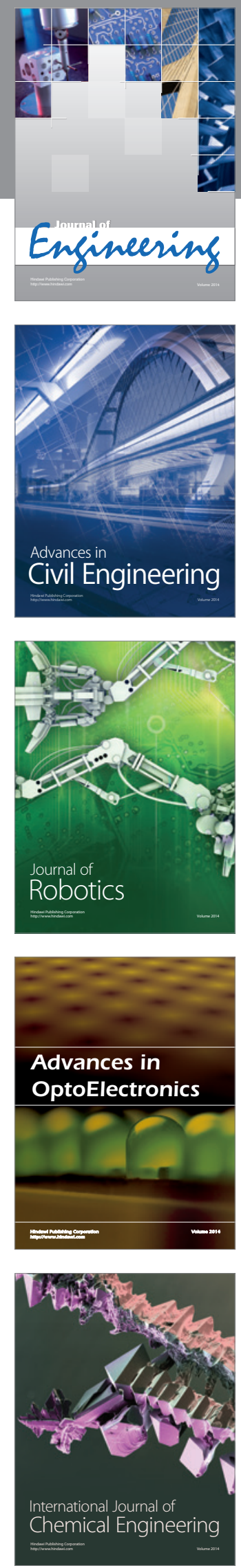

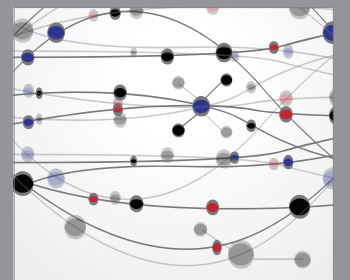

The Scientific World Journal
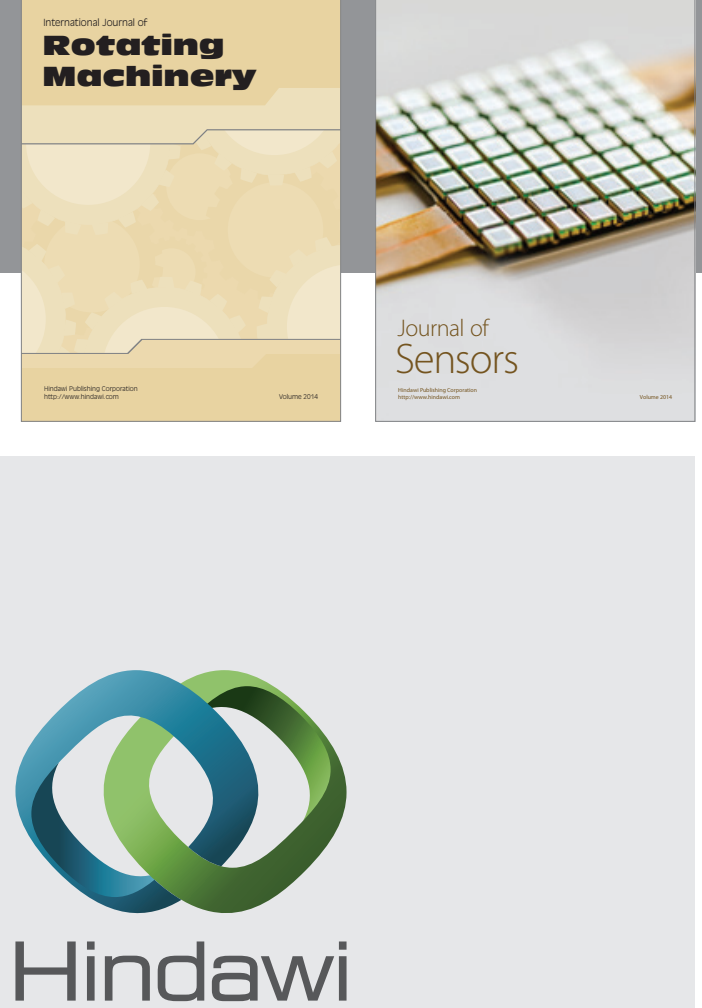

Submit your manuscripts at http://www.hindawi.com
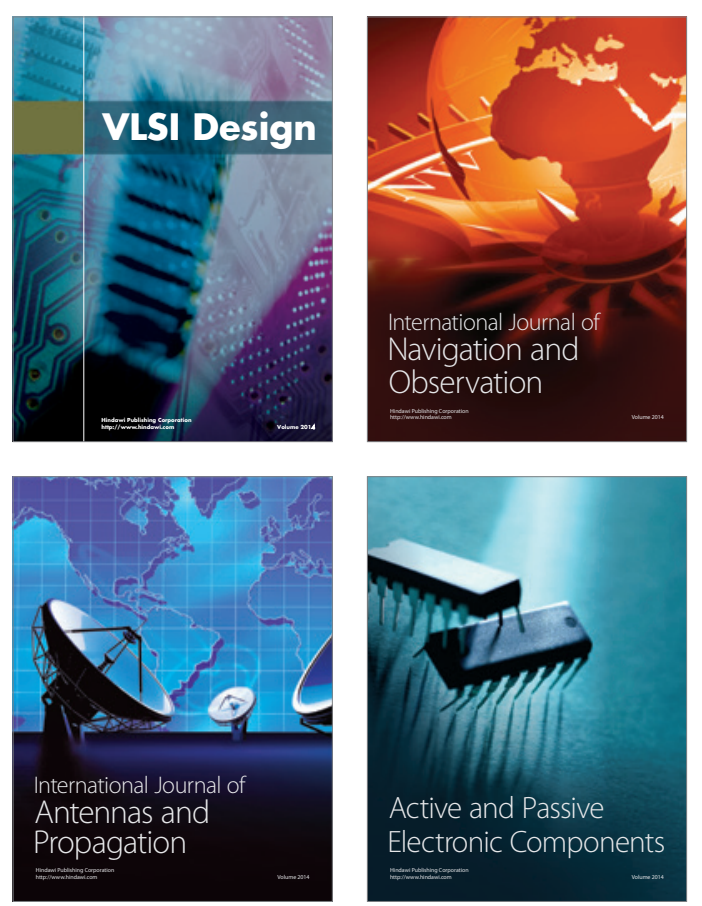
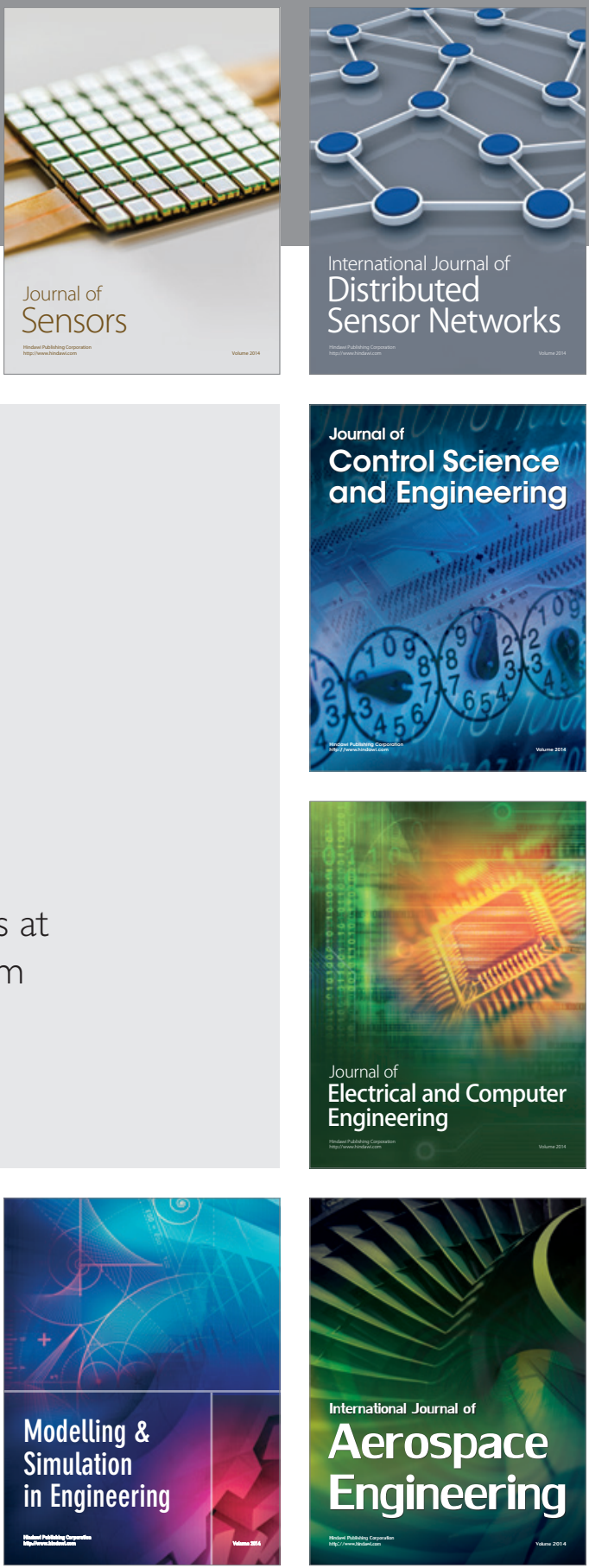

Journal of

Control Science

and Engineering
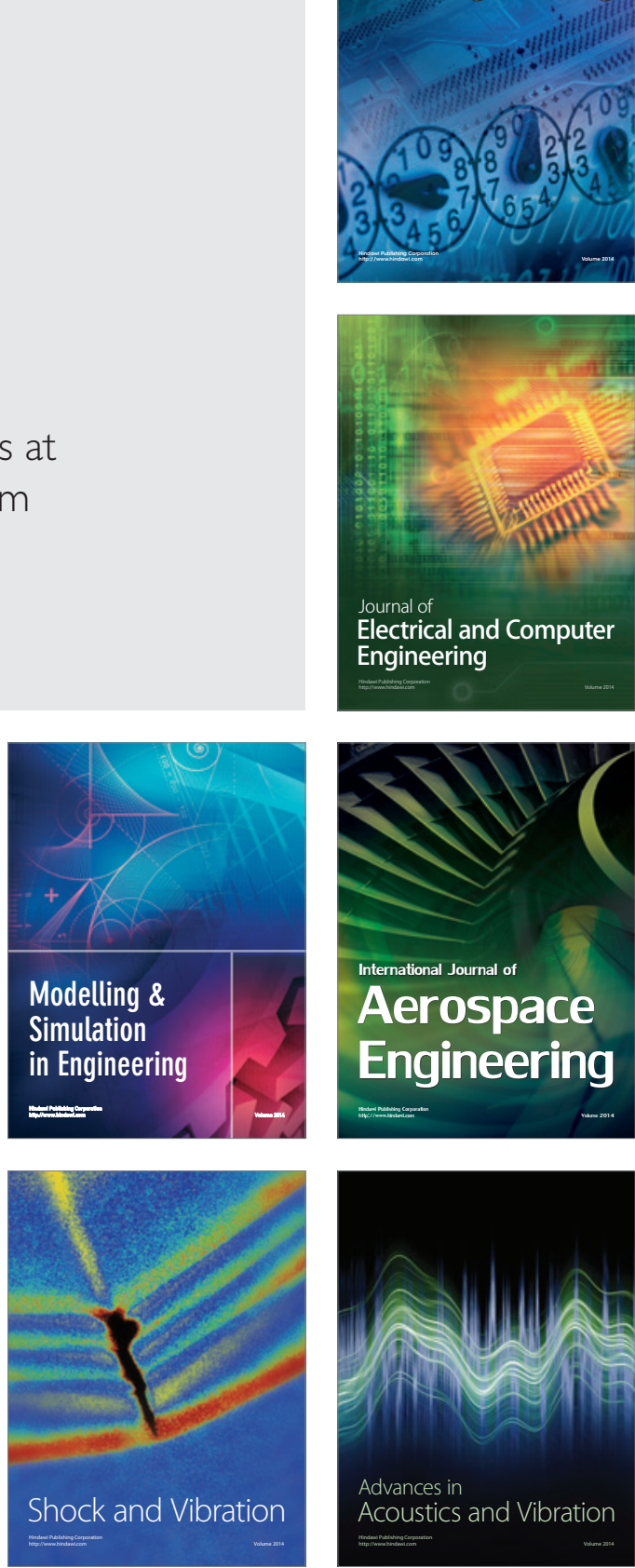